\title{
Automated Anatomical Labeling of Bronchial Branches Extracted from CT Datasets Based on Machine Learning and Combination Optimization and Its Application to Bronchoscope Guidance
}

\author{
Kensaku Mori ${ }^{1,2}$, Shunsuke Ota ${ }^{1}$, Daisuke Deguchi ${ }^{1}$, Takayuki Kitasaka ${ }^{2,3}$, \\ Yasuhito Suenaga $^{1,2}$, Shingo Iwano ${ }^{4}$, Yosihnori Hasegawa ${ }^{2,4}$, \\ Hirotsugu Takabatake ${ }^{5}$, Masaki Mori ${ }^{6}$, and Hiroshi Natori ${ }^{7}$ \\ 1 Graduate School of Information Science, Nagoya University, \\ kensaku@is.nagoya-u.ac.jp \\ 2 Innovative Research Center for Preventive Medical Engineering, Nagoya University \\ 3 Faculty of Management and Information Science, Aichi Institute of Technology \\ ${ }^{4}$ Graduate School of Medicine, Nagoya University \\ 5 Sapporo Minami-Sanjyo Hospital \\ 6 Sapporo Kosei Hospital \\ 7 Nishioka Hospital
}

\begin{abstract}
This paper presents a method for the automated anatomical labeling of bronchial branches extracted from 3D CT images based on machine learning and combination optimization. We also show applications of anatomical labeling on a bronchoscopy guidance system. This paper performs automated labeling by using machine learning and combination optimization. The actual procedure consists of four steps: (a) extraction of tree structures of the bronchus regions extracted from CT images, (b) construction of AdaBoost classifiers, (c) computation of candidate names for all branches by using the classifiers, (d) selection of best combination of anatomical names. We applied the proposed method to 90 cases of 3D CT datasets. The experimental results showed that the proposed method can assign correct anatomical names to $86.9 \%$ of the bronchial branches up to the sub-segmental lobe branches. Also, we overlaid the anatomical names of bronchial branches on real bronchoscopic views to guide real bronchoscopy.
\end{abstract}

\section{Introduction}

A bronchoscope is a flexible endoscope for observing the inside of the bronchus. A chest physician inserts a bronchoscope into the airway through the mouth or the nose to perform a diagnosis, a biopsy, or for treatment. However, the bronchus has a complex tree structure, so physicians easily get disoriented. A system that can guide physicians is strongly expected to be developed. On the other hand, a high resolution $\mathrm{CT}$ has also become available after the release of a 
multi-detector CT scanner. Such CT and bronchoscopic images can be combined, it would be possible to assist bronchoscopic procedures. Several research groups have been working on the development of bronchoscopic navigation systems that utilize pre-operative CT images as maps. If we can overlay the anatomical names of branches currently being observed or paths to target locations [1] on real bronchoscope images, it would be helpful for physicians.

Several research groups have developed automated anatomical labeling procedures for bronchial tree structures 234. Anatomical labeling is performed by comparing input bronchial tree structures with branching pattern models. Kitaoka et al. 2] developed a matching algorithm of branching models and input tree structures using a weighted maximum clique search approach. However, their algorithm does not work well when the tree structures of the input bronchial branches differ from those of model. To cope with this problem, Mori et al. 3] developed a method that can handle variations of branching patterns by introducing multiple branching pattern models. In their method, if we assign anatomical names to a large number of cases, we must also prepare a large number of models to obtain accurate results. Ota presented a method for anatomical labeling based on machine learning [5]. In this method, we considered branch names as categories of the machine learning. However if a mislabeling occurs at a branch, the consequent branches are also mislabeled in the previous method [5]. Such mislabeling often occurs in the right main lobe part of the left lobe part where many branches show similar features in the method [5].

To solve the above problems, we propose a novel approach for anatomical labeling by introducing combination optimization. Classifiers that output branch name candidates with likelihoods are constructed by learning the feature vectors of the bronchial branch names of the learning datasets. The best combination of branch names are obtained under anatomical constraint.

\section{Method}

\subsection{Overview}

Figure 1 shows the processing flow of our proposed method. We construct classifiers that output bronchial branch name candidates with likelihoods using learning datasets. A machine learning approach is utilized here. In the anatomical labeling process, we compute the feature values of bronchial branches extracted from CT images. Then we compute the bronchial branch name candidates with the likelihoods for each bronchial branch using the classifiers. Finally, we determine the anatomical names for each branch by a combination optimization technique. In both the learning and test (labeling) phases, we extract the bronchus regions and the bronchial tree structure from the 3D CT images by Kitasaka's method 6 . The starting point of the tree structure is the trachea. We represent the $i$-th bronchial branch as $\mathbf{b}_{i}(i=1,2, \cdots, N . N$ is the number of bronchial branches.)

For assisting real bronchoscopy, we overlay the following anatomical names of the bronchial branches: (a) where a bronchoscope currently exists; (b) child 


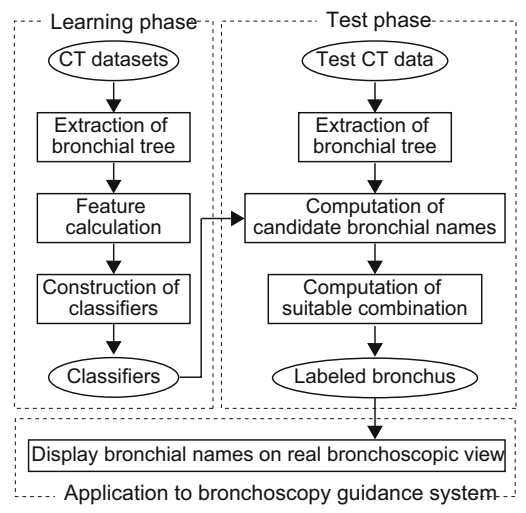

Fig. 1. Flowchart of proposed method

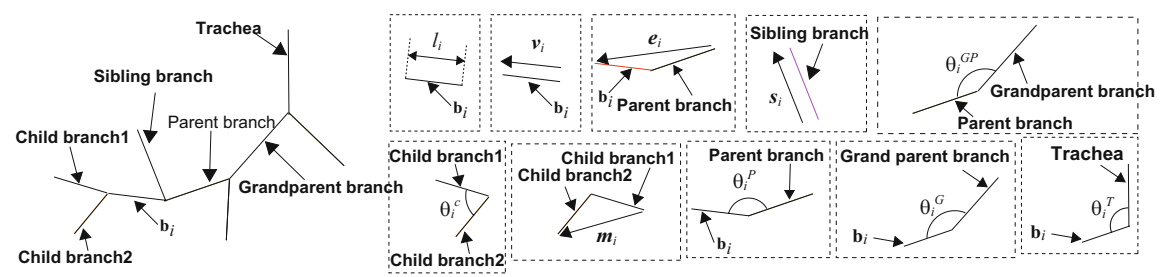

Fig. 2. Ten feature values used in the proposed method

branches of the current branch; and (c) the path to the target point (a set of anatomical names where an bronchoscope should be visited to reach the target point.)

\subsection{Feature Values Computed at Each Bronchial Branch}

We compute the following ten feature values for $\mathbf{b}_{i}$ shown in Fig. 2: (1) $l_{i}$ : length of $\mathbf{b}_{i}$, (2) $\boldsymbol{v}_{i}$ : running direction of $\mathbf{b}_{i}$, (3) $\boldsymbol{m}_{i}$; averaged direction of the running direction of the child branches of $\mathbf{b}_{i},(4) \boldsymbol{e}_{i}$ : relative coordinate of the end point of $\mathbf{b}_{i}$ against the parent branch of $\mathbf{b}_{i}$, (5) $\boldsymbol{s}_{i}$; running direction of sibling branches of $\mathbf{b}_{i}$, (6) $\theta_{i}^{c}$ : angle between child branches of $\mathbf{b}_{i}$, (7) $\theta_{i}^{G}$ : angle between $\mathbf{b}_{i}$ and its grandparent branch, (8) $\theta_{i}^{P}$ : angle between $\mathbf{b}_{i}$ and its parent branch, (9) $\theta_{i}^{G P}$ : angle between grandparent branch of $\mathbf{b}_{i}$ and parent branch of $\mathbf{b}_{i}$, and (10) $\theta_{i}^{T}$ : angle between trachea and $\mathbf{b}_{i}$.

\subsection{Learning Phase (Training of Classifiers)}

Preparation of Learning Datasets. We prepare learning datasets by computing the feature values of the bronchial branches extracted from the CT images. We also manually assign anatomical names to each bronchial branch. 


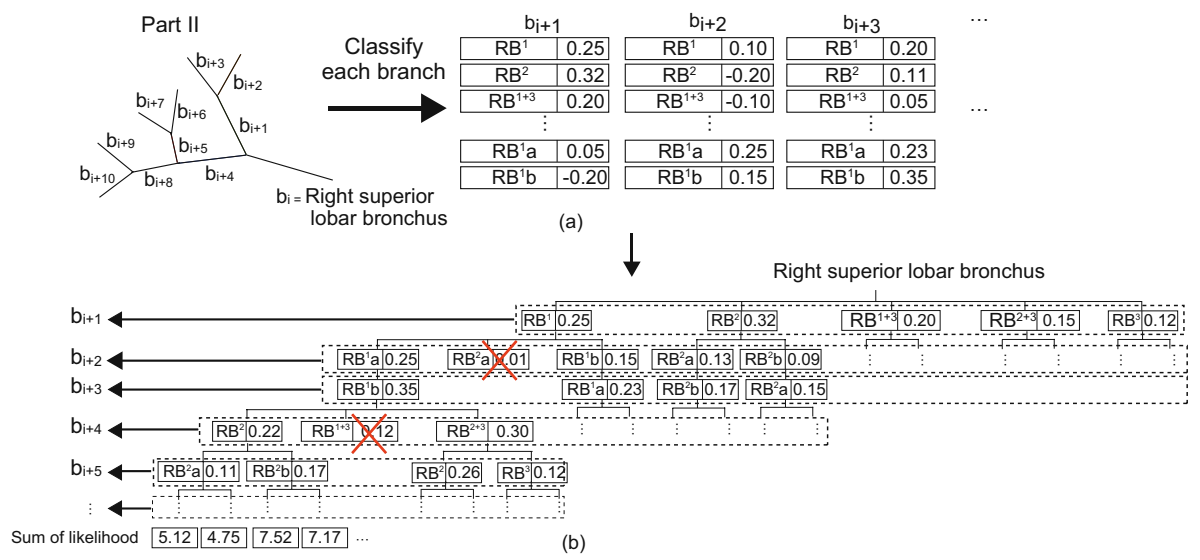

Fig. 3. Illustration of procedures for anatomical labeling of bronchial branches using a combinatorial optimization approach: (a) Example for classifying branches in region II; (b) Example for constructing a combination tree

Construction of Classifiers Using Multi-class AdaBoost. We construct classifiers that output bronchial branch name candidates with likelihoods. These classifiers are constructed using a multi-class AdaBoost technique [7. Thresholding is used as a weak classifier of AdaBoost. We divide the airway tree into six parts: bronchi of: ((i) central area, (ii) right upper lobe part, (iii) right middle lobe part, (iv) right lower lobe part, (v) left upper lobe part, and (vi) left lower lobe part, For each part, classifiers $\mathbf{H}_{\alpha}(\alpha=\mathrm{I}, \cdots, \mathrm{VI})$ are constructed and trained by learning datasets. Part division is performed to reduce the number of branch name combinations computed in the labeling procedure.

\subsection{Test Phase (Anatomical Labeling)}

Generation of List of Bronchial Branch Name Candidates. When we classify bronchial branch $\mathbf{b}_{i}$ existing in part $\alpha$ by classifier $\mathbf{H}_{\alpha}$, the classifier outputs set $\left(\mathcal{B}_{i}^{\alpha}\right)$ of pair $\left(\mathcal{L}_{j}\right)$ of category $\left(L_{j}\right)$ and the likelihood of category $\left(l_{j}\right)$ for branch $\mathbf{b}_{i}$. This process is formulated as

$$
\mathcal{B}_{i}^{\alpha}=\left\{\left(L_{i}^{j}, l_{i}^{j}\right)=\mathcal{L}_{i}^{j}: j=1,2, \cdots, N_{\alpha}\right\}=\mathbf{H}_{\alpha}\left(\mathbf{b}_{i}\right),
$$

where $N_{\alpha}$ shows the number of categories existing in part $\alpha$. The above process is illustrated in Fig. 3(a). In this example, the process generates sets of a pair of the bronchial branch name candidates (categories) and the likelihoods for all bronchial branches existing in part II using classifier $\mathbf{H}_{\mathrm{II}}$.

Construction of Combination Tree. We generate combination tree $\mathcal{T}$ that enumerates the multiple pairs of a bronchial branch name candidate and its likelihood as nodes by

$$
\mathcal{T}=\left\{t_{i}^{j}=\left(L_{i}^{j}, l_{i}^{j}\right): i=1,2, \cdots, W_{j}, j=1,2, \cdots, D\right\}
$$


from the processing results of the previous step. The anatomical constraint of the bronchial tree is considered in the construction of $\mathcal{T}$. Here, we represent the branch name candidate as $L_{i}^{j}$ and its likelihood as $l_{i}^{j}$. Also, we denote the number of nodes at the $j$-th depth of $\mathcal{T}$ and the depth of $\mathcal{T}$ as $D$.

The actual procedure for constructing combination tree $\mathcal{T}$ is explained by Fig. 3(b). First, we construct a combination tree whose root is the origin branch of each part. In the figure, the root branch is $\mathbf{b}_{i}=$ right superior lobar bronchus. Then we traverse the bronchial tree from $\mathbf{b}_{i}$ by depth-first search and add a pair of bronchial branch name candidate and its likelihood at the same depth of $\mathcal{T}$ as the node. In this process, we eliminate nodes added to the combination tree using the rule of the branch names between a parent and a child or siblings. For example, in Fig. 3. $\mathbf{b}_{i+2}$ is a child branch of $\mathbf{b}_{i+1}$, If the branch name candidate of $\mathbf{b}_{i+1}$ is " $\mathrm{RB}^{1}$ (= Rt. B ${ }^{1}$ )", $\mathbf{b}_{i+2}$ cannot be labeled "RB ${ }_{a}^{2}$ ". Hence, we do not add this node. Similarly, $\mathbf{b}_{i+4}$ is a sibling branch of $\mathbf{b}_{i+1}$. If the branch name candidate of $\mathbf{b}_{i+1}$ is " $\mathrm{RB}^{1}$ ", $\mathbf{b}_{i+2}$ cannot be labeled " $\mathrm{RB}^{1+3}$ ". Therefore, we do not add this node. By considering anatomical constraint, computation time can be reduced related to the construction of $\mathcal{T}$.

Anatomical Labeling Using Combination Tree. Anatomical labeling is finally performed by finding path $\mathcal{L}^{*}$ showing the maximum of the sum of the likelihoods along with it in $\mathcal{T}$. This process is formulated as

$$
\mathcal{L}^{*}=\underset{\mathcal{L} \in \mathcal{T}}{\arg \max }\left(l_{i}^{1}+l_{j}^{2}+\cdots+l_{k}^{D}\right)
$$

where path $\mathcal{L}=\left\{t_{i}^{1}, t_{j}^{2}, \cdots, t_{k}^{D}\right\}$ is a set of nodes that exists on a path from the root node to the terminal node.

\subsection{Application to Bronchoscope Guidance System}

We utilize anatomical labeling results to overlay the anatomical names of a branch and its child branches currently being observed on the real bronchoscopic images in the bronchoscopy guidance system. Deguchi's method [8] was used to obtain the branch currently being observed. Furthermore, we compute the insertion path of a bronchoscope by a set of bronchial branch name [3] and overly it on a real bronchoscopic image. During a real bronchoscopy, the bronchoscope location is marked on the path overlaid on real bronchoscopic images. If the bronchoscope deviates off from the pre-planned path, the system shows a "WRONG WAY" message,

\section{Experiments}

We applied the proposed method to 90 cases of 3D CT images. The bronchial regions and branching structures were obtained by Kitasaka's method and manually corrected. To train the classifiers, anatomical names were manually assigned. The number of training data is not sufficient, because there are so many variation 
Table 1. Accuracy of methods in [3, [5] and proposed ones (A) and (B) in each lobe (TR: trachea, LM: left main bronchus, RM: right main bronchus, RU: right upper, RL: tight middle and lower, LU: left upper and LL: left lower lobes)

\begin{tabular}{c||ccccc|c}
\hline \multicolumn{1}{c||}{} & \multicolumn{4}{c}{ Accuracy of segmental } & (subsegmental) & \multicolumn{3}{c}{ branch for each part [\%] } \\
\cline { 2 - 7 } & TR, RB, LB & RU & RM & LU & LL & TOTAL \\
\hline Previous [3] & 100 & 73.6 & 83.5 & 90.5 & 76.7 & 83.5 \\
\hline Previous [5] & $100(100)$ & $86.9(85.2)$ & $89.6(81.7)$ & $98.4(91.6)$ & $87.7(79.0)$ & $91.3(84.3)$ \\
\hline Proposed (a) & $100(100)$ & $81.8(78.2)$ & $93.1(86.2)$ & $98.5(92.6)$ & $89.8(81.1)$ & $92.2(86.9)$ \\
\hline Proposed (b) & $100(100)$ & $81.9(78.9)$ & $85.2(73.0)$ & $91.1(77.2)$ & $79.2(75.1)$ & $85.6(78.6)$ \\
\hline
\end{tabular}
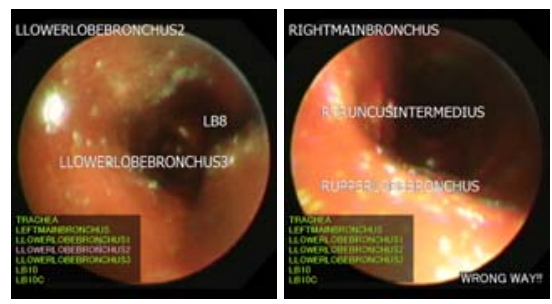

Fig. 4. Application of anatomical labeling in a bronchoscopy guidance system. Cases of inserting a bronchoscope to (right) correct path and (left) incorrect path.

in bronchial branch names. A branch called $\mathrm{LB}^{7}$ rarely exists. Since the number of samples belonging to such categories is very small, this affects the learning of the classifiers, because the feature values obtained from such branches show sparse distribution in the feature space. Therefore, we organized two experiments: (a) adding 100 pairs of samples to all categories generated by normal random and (b) no addition of samples. We used the means and the variances of the feature values of each category as the means and the variances of the normal random. For both cases, we measured the accuracy of the anatomical labeling for branches up to the segmental branches and the sub-segmental branches under the leave-one-out method. For comparison, we also performed the anatomical labeling procedures described in 35. Table 1 shows the labeling results. Here accuracy is computed by (the number of branched correctly labeled) / (the total number of branches)

We utilized the anatomical labeling results in the bronchoscopy guidance system. Figure 4 shows examples of such a bronchoscopy guidance system using an EM tracker. The system shows the bronchial branch names at appropriate positions on the real bronchoscopic images. Figure 4 (right) shows the case where a bronchoscope was inserted into the planned branch and (left) is where the bronchoscope was inserted into the wrong branch.

\section{Discussion and Conclusion}

This paper presented a method for anatomical labeling using combination optimization to precisely assign anatomical names to branches of a large number 


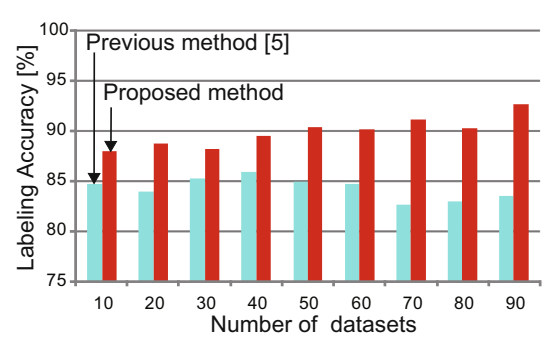

Fig. 5. Comparison of accuracy between previous $\underline{3}$ and proposed methods when number of datasets is increased

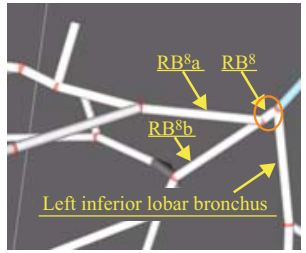

(a)

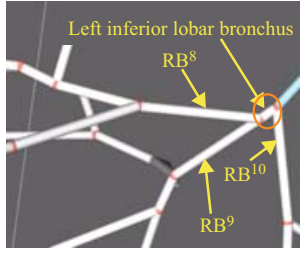

(b)
Fig. 6. Examples of results of anatomical labeling. White lines represent bronchial branches: (a) previous method [9], and (b) proposed one. Underlines show mislabeled branches.

of cases. To confirm the efficacy of the introduction of the machine learning approach in anatomical labeling, we measured the labeling accuracy by changing the number of cases. We randomly selected 10 to 90 cases and calculated the labeling accuracy by the previous method [3. (model-based approach) and the proposed method (machine learning approach.) The results are shown in Fig. 5] In the previous method, labeling accuracy became maximum when the number of cases was 40 and dud not change much for larger cases. In contrast, when we increase the number of cases in the proposed method, the labeling accuracy also increased. As stated in Section 1, we expected to improve the labeling accuracy by such a large number of cases.

Figure [6] shows the labeling results of the method described in [5] and the proposed methods. In this figure, the underlined branch names show incorrectly labeled branches. The branch name having the maximum likelihood of a target branch, which is surrounded by a circle and whose correct name is 'left inferior lobar bronchus' is $\mathrm{RB}^{8}$. Hence, the previous method assigned $\mathrm{RB}^{8}$ to the target branch, and the branches beyond it are mislabeled. On the other hand, the sum of the likelihood for labeling the target branch as 'left inferior lobar bronchus' is higher than that of $\mathrm{RB}^{8}$, so the proposed method was able to correctly label it. As shown in Table 1, the proposed method improved labeling accuracy in most lung part except for RU. Labeling accuracy is significantly improved in RM and LL regions. If a mislabeling occurs at a branch in the previous method [5], the consequent branches were also mislabeled. Such mislabeling often occurred in RM and LL regions where many branches show similar features, On the other hand, the proposed method prevented such mislabeling by combination optimization.

We can see that the labeling accuracy is improved by adding samples artificially generated by normal random because some branches (categories), especially the branches of the right lower and the left lower lobes have a very small amount of samples. In such case, samples are sparsely distributed in the feature space. Consequently the learning process is affected. Further investigation for such cases is needed. 
This paper presented a method for automated anatomical labeling of bronchial branches extracted from 3D CT images. We applied the proposed method to 90 cases of 3D chest CT images. Labeling accuracy was $86.9 \%$ for branches up to the sub-segmental level. Also, we presented a method to display anatomical names on real bronchoscopic images. Future work includes evaluation using more cases.

Acknowledgments. This work is supported by Special Coordination Funds for Promoting Science and Technology by the MEXT, Grant-in-aid for Scientific Research by JSPS and Grant-in-aid for Cancer Research by MHLW, Japan.

\section{References}

1. Kiraly, A.P., Helferty, J.P., Hoffman, E.A., McLennan, G., Higgins, W.E.: ThreeDimensional Path Planning for Virtual Bronchoscopy. IEEE Trans. Med. Imaging 23, 1365-1379 (2004)

2. Kitaoka, H., Park, Y., Tschirren, J., Reinhardt, J.M., Sonka, M., McLennan, G., Hoffman, E.A.: Automated nomenclature labeling of the bronchial tree in 3D-CT lung images. In: Dohi, T., Kikinis, R. (eds.) MICCAI 2002. LNCS, vol. 2489, pp. 1-11. Springer, Heidelberg (2002)

3. Mori, K., Ema, S., Kitasaka, T., Mekada, Y., Ide, I., Murase, H., Suenaga, Y., Takabatake, H., Mori, M., Natori, H.: Automated nomenclature of bronchial branches extracted from CT images and its application to biopsy path planning in virtual bronchoscopy. In: Duncan, J.S., Gerig, G. (eds.) MICCAI 2005. LNCS, vol. 3750, pp. 854-861. Springer, Heidelberg (2005)

4. Tschirren, J., McLennan, G., Palagyi, K., Hoffman, E.A., Sonka, M.: Matching and Anatomical Labeling of Human Airway Tree. IEEE Trans. Med. Imaging 24, 1540 $1547(2005)$

5. Ota, S., Deguchi, D., Kitasaka, T., Mori, K., Suenaga, Y., Hasegawa, Y., Imaizumi, K., Takabatake, H., Mori, M., Natori, H.: Augmented display of anatomical names of bronchial branches for bronchoscopy assistance. In: Dohi, T., Sakuma, I., Liao, H. (eds.) MIAR 2008. LNCS, vol. 5128, pp. 377-384. Springer, Heidelberg (2008)

6. Kitasaka, T., Mori, K., Hasegawa, J., Toriwaki, J.: A Method for Extraction of Bronchus Regions from 3D Chest X-ray CT Images by Analyzing Structural Features of the Bronchus. FORMA 17, 321-338 (2002)

7. Li, L.: Multiclass Boosting with Repartitioning. In: Proc. of the 23rd Int'l Conf. on Machine Learning, pp. 569-576 (2006)

8. Deguchi, D., Ishitani, K., Kitasaka, T., Mori, K., Suenaga, Y., Takabatake, H., Mori, M., Natori, H.: A method for bronchoscope tracking using position sensor without fiducial markers. In: Proc. of SPIE, vol. 6511, p. 65110N-1-12 (2007) 